\section{PSQ-192 MEDICATION REVIEW OF POLYMEDICATED PATIENTS IN A HEALTH MANAGEMENT AREA}

E Recio Sanchez*, V González Rosa, L González Rivas. Hospital Serrania De Ronda, Pharmacy Service, Ronda, Spain

10.1136/ejhpharm-2021-eahpconf.311

Background and importance Polypharmacy is defined as taking more drugs than are clinically necessary and is associated with an increased risk of adverse effects, interactions, less adherence to treatment, more hospitalisations and increased costs. Hence treatment review is important, which allows for a better benefit-risk ratio in the use of medications.

Aim and objectives To analyse the modifications that have been made to polymedicated patients' treatment after review.

Material and methods A cross sectional study was conducted in polymedicated patients (15 or more active prescriptions) selected in a health management area during December 2019. Reviews were carried out by primary care physicians who were previously sent lists with the identifying data of the assigned patients. The pharmacy service held sessions on the strategy to perform to carry out these reviews. Demographic data, associated pathologies and treatment modifications were collected. Deceased patients were excluded.

Results 626 patients were selected in the cross sectional study, $72.5 \%$ (454 patients) were reviewed and of these, 22 patients were excluded due to death. $38.4 \%$ (166) were men and $61.6 \%$ were women (266) with a mean age of 75 years. $55.1 \%$ were 75 years or older. Regarding associated pathologies, 91.4\% had heart disease, 63.0\% diabetes mellitus, 63.9\% dyslipidaemia, $45.6 \%$ respiratory disease (asthma/COPD), $30.1 \%$ chronic kidney failure, $25.7 \%$ atrial fibrillation, $43.3 \%$ had a mental health episode and $10.6 \%$ were oncological. Regarding the number of active prescriptions, 371 patients (85.9\%) had 15-19 prescriptions, 56 patients (13.0\%) had $20-24$, and 5 patients (1.2\%) had more than 25 prescriptions. Modifications were made in $30.1 \%$ of patients: a drug was withdrawn in $87(20.1 \%)$ patients because it was unnecessary, in 3 patients $(0.7 \%)$ due to adverse effects, in 8 patients (1.9\%) due to therapeutic duplication and in $6(1.4 \%)$ due to drug interactions. $20(4.6 \%)$ patients experienced a change in drug dose, $3(0.7 \%)$ patients had the drug changed and 3 $(0.7 \%)$ had a new drug added.

Conclusion and relevance Despite the low number of modifications made, review of polymedicated patients may be useful in our health management area. Most patients treated with more than 15 drugs are over 75 years of age with multiple pathologies, so reduction of prescriptions may help patient safety and improve adherence, which in turn may increase the efficiency of the sanitary system.

\section{REFERENCES AND/OR ACKNOWLEDGEMENTS}

Conflict of interest No conflict of interest

\section{PSQ-193 PATIENT SAFETY IN MEDICATION DISPENSING PERFORMED BY PHARMACONOMISTS: A BEFORE AND AFTER STUDY}

${ }^{1}$ CA Sørensen*, ${ }^{2}$ L Aggergaard, ${ }^{2}$ B Dorthe Krogsgaard. 'Hospital Pharmacy Central Denmark Region, Research and Development, Aarhus N, Denmark; ${ }^{2}$ Hospital Pharmacy Central Denmark Region, Clinical Pharmacy, Randers Nø, Denmark
Background and importance High patient safety is a top priority in healthcare systems worldwide. To secure high patient safety, previous research have focused on different medication concepts (eg, automatic medication dispensing, nurse led medication dispensing or self-administration by patients). However, resources are scarce and decision makers want the most 'value for money'. Therefore, hospital managers investigated the patient safety of medication dispensing performed by pharmaconomists (pharmacy technician with a 3 year degree (180 European credit transfer system points)) compared with nurses.

Aim and objectives To evaluate patient safety of ward level medication dispensing performed by pharmaconomists compared with nurse led medication dispensing.

Material and methods Medication dispensing by pharmaconomists was implemented at seven hospital wards in January 2020. The study was designed as a before and after study. The proportion of ward level dispensing errors was collected through disguised observation of nurses and pharmaconomists in the medicine room before and after implementation of pharmaconomist led medication dispensing. Before data (control group) stem from a $\mathrm{PhD}$ study (by the main author) at the same hospital. These data were collected in 2017-2018 by observing 37 nurses in one ward. After data (intervention group) were collected in March and June 2020. After data were collected in seven wards, to increase the number of pharmaconomists observed (9 instead of only 1) and thus increase generalisability. The collection of after data was performed over two periods due to COVID-19 restrictions at the hospital. A dispensing error was defined as a deviation between the prescription and the dispensed medication (eg, wrong dose). Opportunity for errors (OEs) was defined as any medication dispensed and any medication prescribed but not dispensed. Dispensing error proportion $=($ dispensing errors $/ \mathrm{OEs}) \times 100 \%$.

Results In the control group (before data), 1028 OEs were observed, covering 120 patients. In the intervention group (after data), 1036 OEs were observed, covering 122 patients. The dispensing error proportion was $2.2 \%$ (95\% CI 1.4 to $3.3 \%$ ) in the intervention group (23 errors) compared with 12.8\% (95\% CI 10.9 to 15.0$)$ in the control group (132 errors). The difference between groups was statistically significant $(p=0.00)$.

Conclusion and relevance As pharmaconomists made fewer dispensing errors compared with nurse led medication dispensing, the results indicate high patient safety when medication is dispensed by pharmaconomists. Medication dispensing by pharmaconomists may therefore be a safe alternative to nurse led medication dispensing.

\section{REFERENCES AND/OR ACKNOWLEDGEMENTS}

Conflict of interest No conflict of interest

\section{PSQ-194 MANAGEMENT OF EXPERIMENTAL HEALTH PRODUCTS IN HOSPITAL PHARMACIES: A NATIONAL SURVEY}

${ }^{1} \mathrm{E}$ Delavoipière*, ${ }^{2} \mathrm{C}$ Bouglé, ${ }^{3} \mathrm{M}$ Daouphars, ${ }^{4} \mathrm{~N}$ Donnadieu, ${ }^{5} \mathrm{~L}$ Bernard, ${ }^{6} \mathrm{R}$ Morello, 1) Montreuil, ${ }^{7} \mathrm{~F}$ Divanon, ${ }^{1} \mathrm{~A}$ Alix. 'University Hospital Centre Caen Normandie, Pharmacy, Caen, France; ${ }^{2}$ Omédit, Observatory of Medicines-Medical Devices and Therapeutic Innovation of Normandy, Caen, France; ${ }^{3}$ Cancer Centre Henri Becquerel, Pharmacy, Rouen, France; ${ }^{4}$ University Hospital Centre Rouen Normandie, Pharmacy, Rouen, France; ${ }^{5}$ University Hospital Centre Clermont-Ferrand, Pharmacy, Clermont-Ferrand, France; ${ }^{6}$ University Hospital Centre Caen Normandie, Biostatistics and Clinical Research Unit, Caen, France; ${ }^{7}$ Cancer Centre François Baclesse, Pharmacy, Rouen, France 
Background and importance The management of investigational health products (IHPs) is a major part of conducting clinical trials (CTs). It presents specific risks all along the circuit, with data integrity and patient safety issues. However, few standardised tools are available, and no national inventory has been conducted in hospital pharmacies.

Aim and objectives The aim of this work was to make an inventory of the current situation in our country, and then to prioritise risk reduction standardised tools to develop.

Material and methods A national survey was developed by a regional working group including four clinical research pharmacists (CRPs), a coordinating pharmacist and a pharmacy resident. The 76 questions dealt with the quality approach and the proposal of new tools. The online anonymous survey was emailed to CRPs in health facilities and activated for 2 months.

Results 94 pharmacists participated, allowing a response rate of 70\%: 35 non-university hospitals, 25 university hospital centres, 12 private clinics, 11 cancer centres, 10 not-for-profit private hospitals and 1 academic cancer institute. The results regarding the features of quality approach were: documentation system (76/94), adverse drug event reporting system (77/ 94), prior risk assessment (24/94), training and empowering staff (42/94), using means of evaluation and monitoring (49/ 94, including 13 conducting internal audits) and ISO 9001 certification (10/94). All of these features were synthesised into an overall score: from I (basic quality approach) to IV (ISO certification). Score II was the most frequent (38/94). The score depends on the type of health facility $(\mathrm{p}<0.0005)$ and increases with the number of active CTs $(p<0.0005) .88 /$ 94 pharmacists were interested in standardised tools. All nine proposed tools were useful for over two-thirds of pharmacists. Two tools with the highest utility scores were self-assessment $(\mathrm{p}<0.001)$ and the internal audit grids.

Conclusion and relevance All types of facilities conducting CTs were represented and the response rate suggested an overall interest in this topic of management of IHPs. The quality approach was heterogeneous in hospital pharmacies and depended on the level of activity. The needs identified justify prioritising the self-assessment and traceability audit tools which are being validated for dissemination. Such tools will help to harmonise practices in hospital pharmacies by identifying the specific risks and improving the circuit for IHPs.

References and/or acknowledgements

Conflict of interest No conflict of interest

\section{PSQ-195 IMPACT OF INTENSIFIED CLINICAL DECISION SUPPORT SYSTEMS ON PRESCRIBING ERRORS: AN INTERRUPTED TIME SERIES ANALYSIS IN TAIWAN}

${ }^{1} \mathrm{HY}$ Chen*, ${ }^{2} \mathrm{CH}$ Lee, ${ }^{1} \mathrm{KC}$ Chang, ${ }^{3} \mathrm{WH}$ Chang, ${ }^{4} \mathrm{SC}$ Shao. ${ }^{1}$ Linkou Chang Gung Memorial Hospital, Department of Pharmacy, Taoyuan, Taiwan ROC; ${ }^{2}$ Chang Gung Medical Foundation, Institute of Clinical Pharmacy and Pharmaceutical Sciences, Taoyuan, Taiwan ROC; ${ }^{3}$ Chang Gung Medical Foundation, Department of Pharmaceutical Materials Management Administration Centre, Taoyuan, Taiwan ROC; ${ }^{4}$ Keelung Chang Gung Memorial Hospital, Department of Pharmacy, Keelung, Taiwan ROC

10.1136/ejhpharm-2021-eahpconf.314

Background and importance Clinical decision support systems (CDSSs) are frequently adopted in hospitals to increase prescription accuracy in patients with renal impairment, but the alerts from CDSSs are usually overridden in clinical practice. Therefore, an intensified CDSS with more detailed information should be developed and its effectiveness with regard to prescribing errors determined.

Aim and objectives To evaluate the effectiveness of an intensified CDSS with regard to prescribing errors for inappropriate drugs in cases of renal dysfunction.

Material and methods We conducted a pre- and post-intervention study in an inpatient setting in the largest medical centre in Taiwan in 2019. Previously, the CDSS only reminded clinicians to adjust drugs according to the patient's renal function. After May 2019, we initiated an intensified CDSS directly providing clinicians with the precise drug dosage or alternative drug recommendations for patients with renal impairment. The study outcome was the rate of prescribing errors, as identified by pharmacists after prescribing by clinicians. We used interrupted time series analysis to estimate the trend in prescribing error rate before (January 2019 to May 2019) and after (June 2019 to December 2019) implementing the intensified CDSS. As a control, we conducted similar analyses in another hospital with the same healthcare systems but without an intensified CDSS. We hypothesised there would be a reduction in the rate of prescribing errors for inappropriate drugs in cases of renal dysfunction after the intensification of CDSS in the study hospital, compared with the control hospital.

Results The mean prescribing error rates due to inappropriate drugs in patients with renal dysfunction were 1.70 and 1.59 per 1000 inpatient beds before and after the introduction of the intensified CDSS, respectively, which did not constitute a significant change between the pre- and post- intervention $(\beta=0.01 ; 95 \% \mathrm{CI}-0.32$ to 0.35$)$. However, we observed a non-significantly increased prescribing error rate between the study hospital and the control hospital $(\beta=0.04 ; 95 \% \mathrm{CI}$ -0.51 to 0.59 ) after the introduction of the intensified CDSS. Conclusion and relevance The deployment of an intensified CDSS providing detailed information on how to prescribe drugs for patients with renal impairment may inhibit the increase in prescribing error rate due to inappropriate drugs in patients with renal dysfunction.

\section{REFERENCES AND/OR ACKNOWLEDGEMENTS}

Conflict of interest No conflict of interest

\section{PSQ-196 ASSOCIATION BETWEEN PHOSPHODIESTERASE 5 INHIBITOR USE AND INCIDENT DEMENTIA IN PROSTATE CANCER PATIENTS TREATED WITH ANDROGEN DEPRIVATION THERAPY}

${ }^{1} \mathrm{KC}$ Chang*, ${ }^{2} \mathrm{SC}$ Shao, ${ }^{3} \mathrm{JM}$ Liu, ${ }^{4} \mathrm{ECC}$ Lai. 'Linkou Chang Gung Memorial Hospital, Department of Pharmacy, Taoyuan, Taiwan ROC; ${ }^{2}$ Keelung Chang Gung Memorial Hospital, Department of Pharmacy, Keelung, Taiwan ROC; ${ }^{3}$ Taoyuan General Hospital, Department of Surgery, Taoyuan, Taiwan ROC; ${ }^{4}$ National Cheng Kung University, Institute of Clinical Pharmacy and Pharmaceutical Sciences, Tainan, Taiwan ROC

\subsection{6/ejhpharm-2021-eahpconf.315}

Background and importance Recent studies have indicated that androgen deprivation therapy (ADT) increased the dementia risk in prostate cancer (PCa) patients. Phosphodiesterase 5 inhibitors (PDE5i) with nitric oxide mediated vasodilation can increase blood flow in the brain. However, no current studies have explored the association between PDE5i exposure and dementia in PCa patients treated with ADT. 